\title{
Degenerate binomial coefficients and degenerate hypergeometric functions
}

\author{
Taekyun Kim ${ }^{1,2}$, Dae San Kim ${ }^{3}$ Hyunseok Lee ${ }^{2}$ and Jongkyum Kwon ${ }^{4 *}$
}

\section{"Correspondence:}

mathkjk26@gnu.ac.kr

${ }^{4}$ Department of Mathematics

Education and ERI, Gyeongsang

National University, Jinju, Republic

of Korea

Full list of author information is

available at the end of the article

\begin{abstract}
In this paper, we investigate degenerate versions of the generalized pth order Franel numbers which are certain finite sums involving powers of binomial coefficients. In more detail, we introduce degenerate generalized hypergeometric functions and study degenerate hypergeometric numbers of order $p$. These numbers involve powers of $\lambda$-binomial coefficients and $\lambda$-falling sequence, and can be represented by means of the degenerate generalized hypergeometric functions. We derive some explicit expressions and combinatorial identities for those numbers. We also consider several related special numbers like $\lambda$-hypergeometric numbers of order $p$ and Apostol type $\lambda$-hypergeometric numbers of order $p$, of which the latter reduce in a limiting case to the generalized $p$ th order Franel numbers.
\end{abstract}

MSC: 11B73; 11B83; 33C70

Keywords: Degenerate hypergeometric function; Degenerate bivariate Bell polynomials; Degenerate hypergeometric numbers of order $p ; \lambda$-Hypergeometric numbers of order $p$

\section{Introduction}

First, we study certain finite sums involving powers of binomial coefficients which are called generalized $p$ th order Franel numbers and can be represented in terms of hypergeometric functions. Then, among other things, we find that particular cases of these numbers are connected with many known special numbers and polynomials which include Bernoulli numbers, Euler numbers, Changhee numbers, Daehee numbers, Stirling numbers of the first kind, Catalan numbers, and Legendre polynomials.

In recent years, many mathematicians have devoted their attention to studying various degenerate versions of some special numbers and polynomials $[4,7,8,10,15,16]$. The idea of investigating degenerate versions of some special numbers and polynomials originated from Carlitz's papers [2, 3]. Indeed, he introduced the degenerate Bernoulli and Euler polynomials and numbers, and investigated some arithmetic and combinatorial aspects of them. Here we mention in passing that the degenerate Bernoulli polynomials were later rediscovered by Ustinov under the name of Korobov polynomials of the second kind. Two of the present authors, their colleagues, and some other people have studied quite a few degenerate versions of special numbers and polynomials with their interest

(c) The Author(s) 2020. This article is licensed under a Creative Commons Attribution 4.0 International License, which permits use sharing, adaptation, distribution and reproduction in any medium or format, as long as you give appropriate credit to the original author(s) and the source, provide a link to the Creative Commons licence, and indicate if changes were made. The images or other third party material in this article are included in the article's Creative Commons licence, unless indicated otherwise in a credit line to the material. If material is not included in the article's Creative Commons licence and your intended use is not permitted by statutory regulation or exceeds the permitted use, you will need to obtain permission directly from the copyright holder. To view a copy of this licence, visit http://creativecommons.org/licenses/by/4.0/. 
not only in combinatorial and arithmetic properties but also in differential equations and certain symmetric identities (see $[9,16]$ and the references therein). It is worth noting that this idea of considering degenerate versions of some special polynomials and numbers is not only limited to polynomials but can also be extended to transcendental functions like gamma functions [11, 12]. We believe that studying some degenerate versions of special polynomials and numbers is a very fruitful and promising area of research in which many things remain yet to be uncovered.

Recently, Dolgy and Kim gave some explicit formulas of degenerate Stirling numbers associated with the degenerate special numbers and polynomials. Motivated by Dolgy and Kim's paper [4], we would like to investigate degenerate versions of the generalized $p$ th order Franel numbers. In more detail, we introduce degenerate generalized hypergeometric functions and study degenerate hypergeometric numbers of order $p$. These numbers involve powers of $\lambda$-binomial coefficients and $\lambda$-falling sequence, and can be represented by means of the degenerate generalized hypergeometric functions. We also consider several related special numbers like $\lambda$-hypergeometric numbers of order $p$ and Apostol type $\lambda$-hypergeometric numbers of order $p$, of which the latter reduce in a limiting case to the generalized $p$ th order Franel numbers.

For the rest of this section, we will fix some notations and recall some known results that are needed throughout this paper.

For $\lambda \in \mathbb{R}$, the degenerate exponential function is defined as

$$
e_{\lambda}^{x}(t)=(1+\lambda t)^{\frac{x}{\lambda}}, \quad e_{\lambda}(t)=(1+\lambda t)^{\frac{1}{\lambda}}=e_{\lambda}^{1}(t) \quad(\text { see }[2,3,11,12,14])
$$

From (1), we note that

$$
e_{\lambda}^{x}(t)=\sum_{n=0}^{\infty}(x)_{n, \lambda} \frac{t^{n}}{n !} \quad(\text { see }[4,9-12])
$$

where $(x)_{n, \lambda}$ is the $\lambda$-falling sequence given by

$$
(x)_{0, \lambda}=1, \quad(x)_{n, \lambda}=x(x-\lambda) \cdots(x-(n-1) \lambda) \quad(n \geq 1) .
$$

In [10], the degenerate Stirling numbers of the second kind are defined by

$$
\frac{1}{k !}\left(e_{\lambda}(t)-1\right)^{k}=\sum_{n=k}^{\infty} S_{2, \lambda}(n, k) \frac{t^{n}}{n !} \quad(k \geq 0)
$$

Let

$$
(x)_{0}=1, \quad(x)_{n}=x(x-1)(x-2) \cdots(x-(n-1)) \quad(n \geq 1) .
$$

Then $\lim _{\lambda \rightarrow 0} S_{2, \lambda}(n, k)=S_{2}(n, k)$, where $S_{2}(n, k)$ are the ordinary Stirling numbers of the second kind given by

$$
x^{n}=\sum_{l=0}^{n} S_{2}(n, l)(x)_{l} \quad(n \geq 0) \quad(\text { see }[1-13,15-22]) .
$$


The Stirling numbers of the first kind are defined as

$$
\frac{1}{k !}(\log (1+t))^{k}=\sum_{n=k}^{\infty} S_{1}(n, k) \frac{t^{n}}{n !} \quad(k \geq 0)(\text { see }[13,15]) .
$$

Thus, by (7), we get

$$
(x)_{n}=\sum_{l=0}^{n} S_{1}(n, l) x^{l} \quad(n \geq 0) .
$$

In view of (4), the degenerate Stirling numbers of the first kind are defined by

$$
\frac{1}{k !}\left(\frac{(1+t)^{\lambda}-1}{\lambda}\right)^{k}=\sum_{n=k}^{\infty} S_{1, \lambda}(n, k) \frac{t^{n}}{n !} \quad(\text { see }[4,10]) .
$$

Note that $\lim _{\lambda \rightarrow 0} S_{1, \lambda}(n, k)=S_{1}(n, k)(n, k \geq 0)$.

As is well known, the generalized hypergeometric function $F^{(p, q)}$ is defined by

$$
F^{(p, q)}\left(\begin{array}{c}
a_{1}, a_{2}, \ldots, a_{p} \\
b_{1}, b_{2}, \ldots, b_{q}
\end{array} \mid x\right)=\sum_{k=0}^{\infty} \frac{\left\langle a_{1}\right\rangle_{k} \cdots\left\langle a_{p}\right\rangle_{k}}{\left\langle b_{1}\right\rangle_{k}\left\langle b_{2}\right\rangle_{k} \cdots\left\langle b_{q}\right\rangle_{k}} \frac{x^{k}}{k !}
$$

where $\langle a\rangle_{k}=a(a+1) \cdots(a+(k-1))(k \geq 1),\langle a\rangle_{0}=1($ see $[17,21])$.

For example,

$$
F^{(2,1)}\left(\begin{array}{c}
1, b \\
b
\end{array} \mid-1\right)=\frac{1}{2}, \quad F^{(2,1)}\left(\begin{array}{c}
2, b \\
b
\end{array} \mid-1\right)=\frac{1}{4}, \quad F^{(2,1)}\left(\begin{array}{c}
3, b \\
b
\end{array} \mid-1\right)=\frac{1}{8}, \ldots
$$

The Gauss summation theorem is given by

$$
F^{(2,1)}\left(\begin{array}{c}
a, b \\
c
\end{array} \mid \begin{array}{l}
1
\end{array}\right)=\frac{\Gamma(c) \Gamma(c-a-b)}{\Gamma(c-a) \Gamma(c-b)}
$$

where $R(c)>R(b)>0, R(c-a-b)>0, R(c)>R(a)>0$.

From (11), we note that

$$
F^{(2,1)}\left(\begin{array}{c}
a, b \\
c
\end{array} \mid z\right)=\frac{\Gamma(c)}{\Gamma(b) \Gamma(c-b)} \int_{0}^{1} t^{b-1}(1-t)^{c-b-1}(1-t z)^{-a} d t
$$

where $R(c)>R(b)>0$.

The following are well-known identities related to the binomial coefficients:

$$
\begin{aligned}
& \sum_{k=0}^{n}\left(\begin{array}{l}
n \\
k
\end{array}\right)=2^{n} \quad(n \geq 0), \\
& \sum_{k=0}^{n}(-1)^{k}\left(\begin{array}{l}
n \\
k
\end{array}\right)=0 \quad(n \neq 0, n \in \mathbb{N}), \\
& \sum_{k=0}^{n}\left(\begin{array}{l}
n \\
k
\end{array}\right)^{2}=\frac{(2 n) !}{(n !)^{2}}=\left(\begin{array}{c}
2 n \\
n
\end{array}\right),
\end{aligned}
$$




$$
\begin{aligned}
& \sum_{k=0}^{n}(-1)^{k}\left(\begin{array}{l}
n \\
k
\end{array}\right)^{3}= \begin{cases}0, & \text { if } n \text { is odd, } \\
\frac{(-1)^{n / 2} n !}{\left(\left(\frac{n}{2}\right) !\right)^{2}}, & \text { otherwise, }\end{cases} \\
& \sum_{k=-n}^{n}(-1)^{k}\left(\begin{array}{l}
n+b \\
n+k
\end{array}\right)\left(\begin{array}{l}
n+c \\
c+k
\end{array}\right)\left(\begin{array}{l}
b+c \\
b+k
\end{array}\right)=\frac{\Gamma(b+c+n+1)}{n ! \Gamma(b+1) \Gamma(c+1)} .
\end{aligned}
$$

\section{Sums of powers of $\lambda$-binomial coefficients}

The $\lambda$-binomial coefficients are defined as

$$
\left(\begin{array}{l}
x \\
n
\end{array}\right)_{\lambda}=\frac{(x)_{n, \lambda}}{n !}=\frac{x(x-\lambda) \cdots(x-(n-1) \lambda)}{n !} \quad(n \geq 1), \quad\left(\begin{array}{l}
x \\
0
\end{array}\right)_{\lambda}=1 \quad(\lambda \in \mathbb{R}) .
$$

From (18), we easily get

$$
\left(\begin{array}{c}
x+y \\
n
\end{array}\right)_{\lambda}=\sum_{l=0}^{n}\left(\begin{array}{l}
x \\
l
\end{array}\right)_{\lambda}\left(\begin{array}{c}
y \\
n-l
\end{array}\right)_{\lambda} \quad(n \geq 0) .
$$

By (1), we easily get

$$
B_{\lambda}^{*}(n, k)=\left.\frac{d^{n}}{d t^{n}}\left(e_{\lambda}(t)+1\right)^{k}\right|_{t=0}=\sum_{j=1}^{k}\left(\begin{array}{l}
k \\
j
\end{array}\right)(j)_{n, \lambda}
$$

where $n$ and $k$ are positive integers.

Note that $\lim _{\lambda \rightarrow 0} B_{\lambda}^{*}(n, k)=B(n, k)$, where $B(n, k)$ are defined by Golombek and given by

$$
B(n, k)=\sum_{j=1}^{k}\left(\begin{array}{l}
k \\
j
\end{array}\right) j^{n} \quad(\text { see }[5,6])
$$

Now, we define the degenerate hypergeometric function as

$$
F_{\lambda}\left(\begin{array}{c}
a, b \\
c
\end{array} \mid z\right)=\sum_{n=0}^{\infty} \frac{\langle a\rangle_{n, \lambda}\langle b\rangle_{n, \lambda}}{\langle c\rangle_{n, \lambda}} \frac{z^{n}}{n !}
$$

where $\langle a\rangle_{n, \lambda}=a(a+\lambda) \cdots(a+(n-1) \lambda)(n \geq 1),\langle a\rangle_{0, \lambda}=1$.

From (21), we note that

$$
\begin{aligned}
F_{\lambda}\left(\begin{array}{c}
-n,-n \\
\lambda
\end{array} \mid \lambda e_{\lambda}(t)\right) & =\sum_{k=0}^{\infty} \frac{\langle-n\rangle_{k, \lambda}\langle-n\rangle_{k, \lambda} \lambda^{k}}{\langle\lambda\rangle_{k, \lambda}} \frac{e_{\lambda}^{k}(t)}{k !}=\sum_{k=0}^{\infty} \frac{(n)_{k, \lambda}(n)_{k, \lambda}}{k !} \frac{e_{\lambda}^{k}(t)}{k !} \\
& =\sum_{k=0}^{\infty}\left(\begin{array}{l}
n \\
k
\end{array}\right)_{\lambda}^{2} e_{\lambda}^{k}(t)=\sum_{m=0}^{\infty} \sum_{k=0}^{\infty}\left(\begin{array}{l}
n \\
k
\end{array}\right)_{\lambda}^{2}(k)_{m, \lambda} \frac{t^{m}}{m !},
\end{aligned}
$$

where $n$ is a nonnegative integer.

Let us define

$$
F_{\lambda}\left(\begin{array}{c}
-n,-n \\
\lambda
\end{array} \mid \lambda e_{\lambda}(t)\right)=\sum_{m=0}^{\infty} Q_{\lambda}(m, 2) \frac{t^{m}}{m !} .
$$

Therefore, by (22) and (23), we obtain the following theorem. 
Theorem 2.1 For $m \geq 0$, we have

$$
Q_{\lambda}(m, 2)=\sum_{k=0}^{\infty}\left(\begin{array}{l}
n \\
k
\end{array}\right)_{\lambda}^{2}(k)_{m, \lambda}
$$

We note that $\lim _{\lambda \rightarrow 0} Q_{\lambda}(m, 2)=\sum_{k=0}^{n}\left(\begin{array}{l}n \\ k\end{array}\right)^{2} k^{m}=Q(m, 2)$, which was introduced by Golombek and Marburg (see [5, 6]).

We observe that

$$
F_{\lambda}\left(\begin{array}{c}
-a \\
\times
\end{array} \mid-z\right)=\sum_{k=0}^{\infty}\langle-a\rangle_{k, \lambda} \frac{(-z)^{k}}{k !}=\sum_{k=0}^{\infty} \frac{(a)_{k, \lambda}}{k !} z^{k}=\sum_{k=0}^{\infty}\left(\begin{array}{l}
a \\
k
\end{array}\right)_{\lambda} z^{k}=e_{\lambda}^{a}(z) .
$$

For $n \in \mathbb{N}$, let

$$
F_{\lambda}\left(\begin{array}{c}
-n \\
\times
\end{array} \mid-e_{\lambda}(t)\right)=\left(1+\lambda e_{\lambda}(t)\right)^{\frac{n}{\lambda}}=\sum_{m=0}^{\infty} H_{\lambda}(n, m) \frac{t^{m}}{m !} .
$$

On the one hand, we have

$$
\left(1+\lambda e_{\lambda}(t)\right)^{\frac{n}{\lambda}}=\sum_{k=0}^{\infty}\left(\begin{array}{l}
n \\
k
\end{array}\right)_{\lambda} e_{\lambda}^{k}(t)=\sum_{m=0}^{\infty}\left(\sum_{k=0}^{\infty}\left(\begin{array}{l}
n \\
k
\end{array}\right)_{\lambda}(k)_{m, \lambda}\right) \frac{t^{m}}{m !}
$$

On the other hand, we get

$$
\begin{aligned}
\left(1+\lambda e_{\lambda}(t)\right)^{\frac{n}{\lambda}} & =\left(1+\lambda+\lambda\left(e_{\lambda}(t)-1\right)\right)^{\frac{n}{\lambda}} \\
& =(1+\lambda)^{\frac{n}{\lambda}}\left(1+\frac{\lambda}{1+\lambda}\left(e_{\lambda}(t)-1\right)\right)^{\frac{n}{\lambda}} \\
& =(1+\lambda)^{\frac{n}{\lambda}} \sum_{k=0}^{\infty}\left(\begin{array}{l}
n \\
k
\end{array}\right)_{\lambda}\left(\frac{1}{1+k}\right)^{k}\left(e_{\lambda}(t)-1\right)^{k} \\
& =(1+\lambda)^{\frac{n}{\lambda}} \sum_{k=0}^{\infty}(n)_{k, \lambda}\left(\frac{1}{1+\lambda}\right)^{k} \frac{1}{k !}\left(e_{\lambda}(t)-1\right)^{k} \\
& =\sum_{m=0}^{\infty}\left((1+\lambda)^{\frac{n}{\lambda}} \sum_{k=0}^{m}(n)_{k, \lambda}\left(\frac{1}{1+\lambda}\right)^{k} S_{2, \lambda}(m, k)\right) \frac{t^{m}}{m !} .
\end{aligned}
$$

From (24), (25), and (26), we obtain the following theorem.

Theorem 2.2 For $n \in \mathbb{N}$ and $m \in \mathbb{N} \cup\{0\}$, we have

$$
H_{\lambda}(n, m)=(1+\lambda)^{\frac{n}{\lambda}} \sum_{k=0}^{m}(n)_{k, \lambda}\left(\frac{1}{1+\lambda}\right)^{k} S_{2, \lambda}(m, k)=\sum_{k=0}^{\infty}\left(\begin{array}{l}
n \\
k
\end{array}\right)_{\lambda}(k)_{m, \lambda} .
$$

As is well known, the degenerate Bell polynomials are defined by

$$
e_{\lambda}^{x}\left(e_{\lambda}(t)-1\right)=\sum_{n=0}^{\infty} \operatorname{Bel}_{n, \lambda}(x) \frac{t^{n}}{n !} \quad(\text { see }[16])
$$


By (27), we easily get

$$
\operatorname{Bel}_{n, \lambda}(x)=\sum_{k=0}^{n}(x)_{k, \lambda} S_{2, \lambda}(n, k) \quad(n \geq 0) .
$$

Now, we define the degenerate bivariate Bell polynomials by

$$
e_{\lambda}^{x}\left(y\left(e_{\lambda}(t)-1\right)\right)=\sum_{n=0}^{\infty} \operatorname{Bel}_{n, \lambda}(x, y) \frac{t^{n}}{n !} .
$$

Thus, by (29), we get

$$
\operatorname{Bel}_{n, \lambda}(x, y)=\sum_{k=0}^{n}(x)_{k, \lambda} y^{k} S_{2, \lambda}(n, k) \quad(n \geq 0)
$$

From Theorem 2.2 and (30), we obtain the following corollary.

Corollary 2.3 For $n \in \mathbb{N}$ and $m \in \mathbb{N} \cup\{0\}$, we have

$$
H_{\lambda}(n, m)=(1+\lambda)^{\frac{n}{\lambda}} \operatorname{Bel}_{m, \lambda}\left(n, \frac{1}{1+\lambda}\right) .
$$

Note that

$$
\lim _{\lambda \rightarrow 0} H_{\lambda}(n, m)=\sum_{k=0}^{\infty} \frac{n^{k}}{k !} k^{m}=e^{n} \sum_{k=0}^{m} n^{k} S_{2}(m, k) .
$$

We observe that

$$
\begin{aligned}
H_{\lambda}(n, 1) & =\sum_{k=0}^{\infty}\left(\begin{array}{l}
n \\
k
\end{array}\right)_{\lambda}(k)_{1, \lambda}=\sum_{k=1}^{\infty} \frac{(n)_{k, \lambda}}{(k-1) !} \\
& =\sum_{k=0}^{\infty}\left(\begin{array}{l}
n \\
k
\end{array}\right)_{\lambda}(n-k \lambda)=n \sum_{k=0}^{\infty}\left(\begin{array}{l}
n \\
k
\end{array}\right)_{\lambda}-\lambda \sum_{k=0}^{\infty}\left(\begin{array}{l}
n \\
k
\end{array}\right)_{\lambda} k \\
& =n \sum_{k=0}^{\infty}\left(\begin{array}{l}
n \\
k
\end{array}\right)_{\lambda}-\lambda H_{\lambda}(n, 1) .
\end{aligned}
$$

Thus, by (31), we get

$$
(1+\lambda) H_{\lambda}(n, 1)=n \sum_{k=0}^{\infty}\left(\begin{array}{l}
n \\
k
\end{array}\right)_{\lambda}=n(1+\lambda)^{\frac{n}{\lambda}} .
$$

From (32), we have

$$
H_{\lambda}(n, 1)=n(1+\lambda)^{\frac{n}{\lambda}-1} \quad(\lambda \neq-1) .
$$


For $m=2$, we have

$$
\begin{aligned}
\sum_{k=0}^{\infty}\left(\begin{array}{l}
n \\
k
\end{array}\right)_{\lambda}(k)_{2, \lambda} & =\sum_{k=0}^{\infty} \frac{(n)_{k, \lambda}}{k !} k(k-\lambda)=\sum_{k=1}^{\infty} \frac{(n)_{k, \lambda}}{(k-1) !}(k-\lambda) \\
& =\sum_{k=1}^{\infty} \frac{(n)_{k, \lambda}}{(k-1) !}(k-1+1-\lambda)=\sum_{k=2}^{\infty} \frac{(n)_{k, \lambda}}{(k-2) !}+(1-\lambda) \sum_{k=1}^{\infty} \frac{(n)_{k, \lambda}}{(k-1) !} \\
& =\sum_{k=2}^{\infty} \frac{(n-2 \lambda)_{k-2, \lambda}}{(k-2) !} n(n-\lambda)+(1-\lambda) n \sum_{k=1}^{\infty} \frac{(n-\lambda)_{k-1, \lambda}}{(k-1) !} \\
& =n(n-\lambda) \sum_{k=2}^{\infty}\left(\begin{array}{c}
n-2 \lambda \\
k-2
\end{array}\right)_{\lambda}+n(1-\lambda) \sum_{k=1}^{\infty}\left(\begin{array}{c}
n-\lambda \\
k-1
\end{array}\right)_{\lambda} \\
& =n(n-\lambda) \sum_{k=0}^{\infty}\left(\begin{array}{c}
n-2 \lambda \\
k
\end{array}\right)_{\lambda}+n(1-\lambda) \sum_{k=0}^{\infty}\left(\begin{array}{c}
n-\lambda \\
k
\end{array}\right)_{\lambda} \\
& =n(n-\lambda)(1+\lambda)^{\frac{n}{\lambda}-2}+n(1-\lambda)(1+\lambda)^{\frac{n}{\lambda}-1} \\
& =n\left(n+1-\lambda-\lambda^{2}\right)(1+\lambda)^{\frac{n}{\lambda}-2} .
\end{aligned}
$$

By (33), we get

$$
H_{\lambda}(n, 2)=n(n+1-2 \lambda)(1+\lambda)^{\frac{n}{\lambda}-2} .
$$

Let us take $m=3$. Then we have

$$
\begin{aligned}
H_{\lambda}(n, 3)= & \sum_{k=0}^{\infty}\left(\begin{array}{l}
n \\
k
\end{array}\right)_{\lambda}(k)_{3, \lambda}=\sum_{k=0}^{\infty}\left(\begin{array}{l}
n \\
k
\end{array}\right)_{\lambda} k(k-\lambda)(k-2 \lambda) \\
= & \sum_{k=1}^{\infty} \frac{(n)_{k, \lambda}}{(k-1) !}(k-\lambda)(k-2 \lambda)=\sum_{k=1}^{\infty} \frac{(n)_{k, \lambda}}{(k-1) !}(k-1+1-\lambda)(k-2 \lambda) \\
= & \sum_{k=2}^{\infty} \frac{(n)_{k, \lambda}}{(k-2) !}(k-2 \lambda)+(1-\lambda) \sum_{k=1}^{\infty} \frac{(n)_{k, \lambda}}{(k-1) !}(k-2 \lambda) \\
= & \sum_{k=3}^{\infty} \frac{(n)_{k, \lambda}}{(k-3) !}+3(1-\lambda) \sum_{k=2}^{\infty} \frac{(n)_{k, \lambda}}{(k-2) !}+(1-\lambda)(1-2 \lambda) \sum_{k=1}^{\infty} \frac{(n)_{k, \lambda}}{(k-1) !} \\
= & (n)_{3, \lambda} \sum_{k=0}^{\infty}\left(\begin{array}{c}
n-3 \lambda \\
k
\end{array}\right)_{\lambda}+3(1-\lambda)(n)_{2, \lambda} \sum_{k=0}^{\infty}\left(\begin{array}{c}
n-2 \lambda \\
k
\end{array}\right)_{\lambda} \\
& +(1-\lambda)(1-2 \lambda)(n)_{1, \lambda} \sum_{k=0}^{\infty}\left(\begin{array}{c}
n-\lambda \\
k
\end{array}\right)_{\lambda} \\
= & (n)_{3, \lambda}(1+\lambda)^{\frac{n}{\lambda}-3}+3(1-\lambda)(n)_{2, \lambda}(1+\lambda)^{\frac{n}{\lambda}-2}+(1-\lambda)(1-2 \lambda) n(1+\lambda)^{\frac{n}{\lambda}-1} .
\end{aligned}
$$

Note that

$$
\lim _{\lambda \rightarrow 1} H_{\lambda}(n, 3)=\sum_{k=0}^{n}\left(\begin{array}{l}
n \\
k
\end{array}\right)(k)_{3}=(n)_{3} 2^{n-3}
$$


and

$$
\lim _{\lambda \rightarrow 0} H_{\lambda}(n, 3)=\sum_{k=0}^{\infty} \frac{n^{k}}{k !} k^{3}=n\left(n^{2}+3 n+1\right) e^{n}
$$

For $s \in \mathbb{C}$ with $R(s)>0$, the gamma function is defined by

$$
\Gamma(s)=\int_{0}^{\infty} e^{-t} t^{s-1} d t
$$

Let $n$ be a nonnegative integer. Then

$$
\frac{\langle b\rangle_{n, \lambda}}{\langle c\rangle_{n, \lambda}}=\frac{\Gamma\left(\frac{b}{\lambda}+n\right) \Gamma\left(\frac{c}{\lambda}\right)}{\Gamma\left(\frac{c}{\lambda}+n\right) \Gamma\left(\frac{b}{\lambda}\right)}
$$

where $R\left(\frac{c}{\lambda}\right)>0$ and $R\left(\frac{b}{\lambda}\right)>0$.

For $R\left(\frac{c}{\lambda}\right)>R\left(\frac{b}{\lambda}\right)>0$, we have

$$
\frac{\Gamma\left(\frac{b}{\lambda}+n\right) \Gamma\left(\frac{c}{\lambda}-\frac{b}{\lambda}\right)}{\Gamma\left(\frac{c}{\lambda}+n\right)}=\int_{0}^{1} t^{\frac{b}{\lambda}+n-1}(1-t)^{\frac{c}{\lambda}-\frac{b}{\lambda}-1} d t
$$

From (35), we note that

$$
\begin{aligned}
F_{\lambda}\left(\begin{array}{c}
a, b \\
c
\end{array} \mid z\right) & =\sum_{n=0}^{\infty} \frac{\langle a\rangle_{n, \lambda}\langle b\rangle_{n, \lambda}}{\langle c\rangle_{n, \lambda}} \frac{z^{n}}{n !} \\
& =\frac{\Gamma\left(\frac{c}{\lambda}\right)}{\Gamma\left(\frac{b}{\lambda}\right) \Gamma\left(\frac{c}{\lambda}-\frac{b}{\lambda}\right)} \int_{0}^{1} t^{\frac{b}{\lambda}-1}(1-t)^{\frac{c}{\lambda}-\frac{b}{\lambda}-1}(1-\lambda t z)^{-\frac{a}{\lambda}} d t .
\end{aligned}
$$

In particular, for $z=\frac{1}{\lambda}(\lambda \neq 0)$, from (11) we get

$$
F_{\lambda}\left(\begin{array}{c|c}
a, b & \frac{1}{\lambda} \\
c & \lambda
\end{array}\right)=\frac{\Gamma\left(\frac{c}{\lambda}\right) \Gamma\left(\frac{c}{\lambda}-\frac{b}{\lambda}-\frac{a}{\lambda}\right)}{\Gamma\left(\frac{c}{\lambda}-\frac{b}{\lambda}\right) \Gamma\left(\frac{c}{\lambda}-\frac{a}{\lambda}\right)}, \quad \text { where } R\left(\frac{c}{\lambda}-\frac{b}{\lambda}-\frac{a}{\lambda}\right)>0
$$

For $n \in \mathbb{N}$, by (37), we get

$$
F_{\lambda}\left(\begin{array}{c|c}
-n,-n & \frac{1}{\lambda} \\
\lambda & \lambda
\end{array}\right)=\frac{\Gamma(1) \Gamma\left(1+\frac{2 n}{\lambda}\right)}{\Gamma\left(1+\frac{n}{\lambda}\right) \Gamma\left(1+\frac{n}{\lambda}\right)}=\frac{2 \lambda}{n} \frac{\Gamma\left(\frac{2 n}{\lambda}\right)}{\left(\Gamma\left(\frac{n}{\lambda}\right)\right)^{2}}
$$

where $\lambda$ is a positive real number.

On the other hand,

$$
F_{\lambda}\left(\begin{array}{c|c}
-n,-n & \frac{1}{\lambda} \\
\lambda & \lambda
\end{array}\right)=\sum_{k=0}^{\infty} \frac{\langle-n\rangle_{k, \lambda}\langle-n\rangle_{k, \lambda}}{\langle\lambda\rangle_{k, \lambda}} \frac{\left(\frac{1}{\lambda}\right)^{k}}{k !}=\sum_{k=0}^{\infty} \lambda^{-2 k}\left(\begin{array}{l}
n \\
k
\end{array}\right)_{\lambda}^{2} .
$$

Therefore, by (38) and (39), we obtain the following theorem. 
Theorem 2.4 Let $\lambda$ be a positive real number. For $n \in \mathbb{N}$, we have

$$
\sum_{k=0}^{\infty} \lambda^{-2 k}\left(\begin{array}{l}
n \\
k
\end{array}\right)_{\lambda}^{2}=\frac{2 \lambda}{n} \frac{\Gamma\left(\frac{2 n}{\lambda}\right)}{\left(\Gamma\left(\frac{n}{\lambda}\right)\right)^{2}}
$$

Note that

$$
\lim _{\lambda \rightarrow 1} \sum_{k=0}^{\infty} \lambda^{-2 k}\left(\begin{array}{l}
n \\
k
\end{array}\right)_{\lambda}^{2}=\sum_{k=0}^{n}\left(\begin{array}{l}
n \\
k
\end{array}\right)^{2}=\frac{(2 n) !}{(n !)^{2}}=\left(\begin{array}{c}
2 n \\
n
\end{array}\right) .
$$

Now, we define the degenerate generalized hypergeometric function as

$$
F_{\lambda}^{(p, q)}\left(\begin{array}{c}
a_{1}, a_{2}, \ldots, a_{p} \\
b_{1}, \ldots, b_{q}
\end{array} \mid z\right)=\sum_{k=0}^{\infty} \frac{\left\langle a_{1}\right\rangle_{k, \lambda} \cdots\left\langle a_{p}\right\rangle_{k, \lambda}}{\left\langle b_{1}\right\rangle_{k, \lambda} \cdots\left\langle b_{q}\right\rangle_{k, \lambda}} \frac{z^{k}}{k !}, \quad \text { where }|z|<1
$$

Let $n$ be a positive integer. Then we define the degenerate hypergeometric numbers of order $p$ by

$$
F_{\lambda}^{(p, p-1)}\left(\begin{array}{c}
-n-n, \ldots,-n \\
\lambda, \ldots, \lambda
\end{array} \mid(-1)^{p} \lambda^{p-1} e_{\lambda}(t)\right)=\sum_{m=0}^{\infty} H_{\lambda}^{(p)}(n, m) \frac{t^{m}}{m !} .
$$

From (23) and (24), we note that $H_{\lambda}(n, m)=H_{\lambda}^{(1)}(n, m)$, and $Q_{\lambda}(m, 2)=H_{\lambda}^{(2)}(n, m)$.

In (40), we note that

$$
\begin{aligned}
& F_{\lambda}^{(p, p-1)}\left(\begin{array}{c}
-n,-n, \ldots,-n \\
\lambda, \ldots, \lambda
\end{array} \mid(-1)^{p} \lambda^{p-1} e_{\lambda}(t)\right) \\
& =\sum_{k=0}^{\infty} \frac{\langle-n\rangle_{k, \lambda}\langle-n\rangle_{k, \lambda} \cdots\langle-n\rangle_{k, \lambda}}{\langle\lambda\rangle_{k, \lambda}\langle\lambda\rangle_{k, \lambda} \cdots\langle\lambda\rangle_{k, \lambda}} \frac{(-1)^{p k} \lambda^{(p-1) k}}{k !} e_{\lambda}^{k}(t) \\
& =\sum_{k=0}^{\infty} \frac{\left((n)_{k, \lambda}\right)^{p}}{(k !)^{p-1}} \frac{e_{\lambda}^{k}(t)}{k !}=\sum_{k=0}^{\infty}\left(\begin{array}{l}
n \\
k
\end{array}\right)_{\lambda}^{p} e_{\lambda}^{k}(t) \\
& =\sum_{m=0}^{\infty}\left(\sum_{k=0}^{\infty}\left(\begin{array}{l}
n \\
k
\end{array}\right)_{\lambda}^{p}(k)_{m, \lambda}\right) \frac{t^{m}}{m !} .
\end{aligned}
$$

Therefore, by (41) and (42), we obtain the following theorem.

Theorem 2.5 For $n, p \in \mathbb{N}$ and $m \in \mathbb{N} \cup\{0\}$, we have

$$
H_{\lambda}^{(p)}(n, m)=\sum_{k=0}^{\infty}\left(\begin{array}{l}
n \\
k
\end{array}\right)_{\lambda}^{p}(k)_{m, \lambda}
$$

Note that

$$
\lim _{\lambda \rightarrow 1} H_{\lambda}^{(p)}(n, m)=\sum_{k=0}^{n}\left(\begin{array}{l}
n \\
k
\end{array}\right)^{p}(k)_{m} \quad \text { and } \quad \lim _{\lambda \rightarrow 0} H_{\lambda}^{(p)}(n, m)=\sum_{k=0}^{\infty} \frac{n^{k p}}{(k !)^{p}} k^{m} .
$$


From (42), we note that

$$
\begin{aligned}
& F_{\lambda}^{(p, p-1)}\left(\begin{array}{c}
-n,-n, \ldots,-n \\
\lambda, \ldots, \lambda
\end{array} \mid(-1)^{p} \lambda^{p-1} e_{\lambda}(t)\right) \\
& =\sum_{k=0}^{\infty}\left(\begin{array}{l}
n \\
k
\end{array}\right)_{\lambda}^{p} e_{\lambda}^{k}(t) \\
& =\sum_{k=0}^{\infty}\left(\begin{array}{l}
n \\
k
\end{array}\right)_{\lambda}^{p} \sum_{l=0}^{k}\left(\begin{array}{l}
k \\
l
\end{array}\right)\left(e_{\lambda}(t)-1\right)^{l}=\sum_{k=0}^{\infty}\left(\begin{array}{l}
n \\
k
\end{array}\right)_{\lambda}^{p} \sum_{l=0}^{k}(k)_{l} \sum_{m=l}^{\infty} S_{2, \lambda}(m, l) \frac{t^{m}}{m !} \\
& =\sum_{m=0}^{\infty}\left(\sum_{k=0}^{\infty} \sum_{l=0}^{k}\left(\begin{array}{l}
n \\
k
\end{array}\right)_{\lambda}^{p}(k)_{l} S_{2, \lambda}(m, l)\right) \frac{t^{m}}{m !} .
\end{aligned}
$$

Therefore, by (41) and (43), we obtain the following theorem.

Theorem 2.6 For $n, p \in \mathbb{N}$ and $m \in \mathbb{N} \cup\{0\}$, we have

$$
H_{\lambda}^{(p)}(n, m)=\sum_{k=0}^{\infty} \sum_{l=0}^{k}\left(\begin{array}{l}
n \\
k
\end{array}\right)_{\lambda}^{p}(k)_{l} S_{2, \lambda}(m, l)
$$

By (41) and (42), we get

$$
H_{\lambda}^{(p)}(n, 0)=\sum_{k=0}^{\infty}\left(\begin{array}{l}
n \\
k
\end{array}\right)_{\lambda}^{p}=F_{\lambda}^{(p, p-1)}\left(\begin{array}{c}
-n,-n, \ldots,-n \\
\lambda, \ldots, \lambda
\end{array} \mid(-1)^{p} \lambda^{p-1}\right) .
$$

Note that

$$
\sum_{k=0}^{n}\left(\begin{array}{l}
n \\
k
\end{array}\right)^{p}=\lim _{\lambda \rightarrow 1} H_{\lambda}^{(p)}(n, 0)=F^{(p, p-1)}\left(\begin{array}{c}
-n,-n, \ldots,-n \\
1, \ldots, 1
\end{array} \mid(-1)^{p}\right) .
$$

From (40), we have

$$
\begin{aligned}
& F_{\lambda}^{(p, p-1)}\left(\begin{array}{c}
-n,-n, \ldots,-n \\
\lambda, \ldots, \lambda
\end{array} \mid(-\lambda)^{p-1} e_{\lambda}(t)\right) \\
& \quad=\sum_{k=0}^{\infty}\left(\begin{array}{l}
n \\
k
\end{array}\right)_{\lambda}^{p}(-1)^{k} e_{\lambda}^{k}(t)=\sum_{m=0}^{\infty}\left(\sum_{k=0}^{\infty}\left(\begin{array}{l}
n \\
k
\end{array}\right)_{\lambda}^{p}(-1)^{k}(k)_{m, \lambda}\right) \frac{t^{m}}{m !} .
\end{aligned}
$$

Thus, by (44), we get

$$
F_{\lambda}^{(p, p-1)}\left(\begin{array}{c}
-n,-n, \ldots,-n \\
\lambda, \ldots, \lambda
\end{array} \mid(-\lambda)^{p-1}\right)=\sum_{k=0}^{\infty}\left(\begin{array}{l}
n \\
k
\end{array}\right)_{\lambda}^{p}(-1)^{k}
$$


Note that

$$
\begin{aligned}
& \lim _{\lambda \rightarrow 1} F_{\lambda}^{(p, p-1)}\left(\begin{array}{c}
-n,-n, \ldots,-n \\
\lambda, \ldots, \lambda
\end{array} \mid(-\lambda)^{p-1}\right) \\
& \quad=\sum_{k=0}^{n}\left(\begin{array}{l}
n \\
k
\end{array}\right)^{p}(-1)^{k}=F^{(p, p-1)}\left(\begin{array}{c}
-n,-n, \ldots,-n \\
1, \ldots, 1
\end{array} \mid(-1)^{p-1}\right) .
\end{aligned}
$$

\section{Further remarks}

Let $n$ be a positive integer. From (10), we have

$$
\begin{aligned}
F^{(2,1)}\left(\begin{array}{c}
-n,-n \\
1
\end{array} \mid e_{\lambda}(t)\right) & =\sum_{k=0}^{\infty} \frac{\langle-n\rangle_{k}\langle-n\rangle_{k}}{\langle 1\rangle_{k}} \frac{e_{\lambda}^{k}(t)}{k !} \\
& =\sum_{k=0}^{n}\left(\begin{array}{l}
n \\
k
\end{array}\right)^{2} e_{\lambda}^{k}(t)=\sum_{m=0}^{\infty}\left(\sum_{k=0}^{n}\left(\begin{array}{l}
n \\
k
\end{array}\right)^{2}(k)_{m, \lambda}\right) \frac{t^{m}}{m !} .
\end{aligned}
$$

Now, we define the $\lambda$-hypergeometric numbers of order $p$ by

$$
F^{(p, p-1)}\left(\begin{array}{c}
-n,-n, \ldots,-n \\
1, \ldots, 1
\end{array} \mid(-1)^{p} e_{\lambda}(t)\right)=\sum_{m=0}^{\infty} H_{m, \lambda}^{(p)}(n) \frac{t^{m}}{m !}
$$

By (46) and (47), we get

$$
H_{m, \lambda}^{(2)}(n)=\sum_{k=0}^{n}\left(\begin{array}{l}
n \\
k
\end{array}\right)^{2}(k)_{m, \lambda}
$$

where $n \in \mathbb{N}$ and $m \in \mathbb{N} \cup\{0\}$.

The alternating $\lambda$-hypergeometric numbers of order $p$ are defined by

$$
F^{(p, p-1)}\left(\begin{array}{c}
-n,-n, \ldots,-n \\
1, \ldots, 1
\end{array} \mid(-1)^{p-1} e_{\lambda}(t)\right)=\sum_{m=0}^{\infty} T_{m, \lambda}^{(p)}(n) \frac{t^{m}}{m !}
$$

By (10), we get

$$
F^{(2,1)}\left(\begin{array}{c}
-n,-n \\
1
\end{array} \mid-e_{\lambda}(t)\right)=\sum_{m=0}^{\infty}\left(\sum_{k=0}^{\infty}\left(\begin{array}{l}
n \\
k
\end{array}\right)^{2}(-1)^{k}(k)_{m, \lambda}\right) \frac{t^{m}}{m !} .
$$

From (49) and (50), we have

$$
T_{m, \lambda}^{(2)}(n)=\sum_{k=0}^{n}\left(\begin{array}{l}
n \\
k
\end{array}\right)^{2}(-1)^{k}(k)_{m, \lambda}
$$

where $m \in \mathbb{N} \cup\{0\}$ and $n \in \mathbb{N}$.

In general, we have

$$
T_{m, \lambda}^{(p)}(n)=\sum_{k=0}^{n}\left(\begin{array}{l}
n \\
k
\end{array}\right)^{p}(-1)^{k}(k)_{m, \lambda} \quad \text { and } \quad H_{m, \lambda}^{(p)}(n)=\sum_{k=0}^{n}\left(\begin{array}{l}
n \\
k
\end{array}\right)^{p}(k)_{m, \lambda},
$$

where $n, p \in \mathbb{N}$ and $m \in \mathbb{N} \cup\{0\}$. 
We observe that

$$
F^{(1,0)}\left(\begin{array}{c}
-n \\
\times
\end{array} \mid-e_{\lambda}(t)\right)=\sum_{k=0}^{n}\left(\begin{array}{l}
n \\
k
\end{array}\right) e_{\lambda}^{k}(t)
$$

and

$$
F^{(1,0)}\left(\begin{array}{c}
-n \\
\times
\end{array} \mid e_{\lambda}(t)\right)=\sum_{k=0}^{n}\left(\begin{array}{l}
n \\
k
\end{array}\right)(-1)^{k} e_{\lambda}^{k}(t)
$$

Thus, we note that

$$
H_{m, \lambda}^{(1)}(n)=\sum_{k=0}^{n}\left(\begin{array}{l}
n \\
k
\end{array}\right)(k)_{m, \lambda}, \quad T_{m, \lambda}^{(1)}=\sum_{k=0}^{n}\left(\begin{array}{l}
n \\
k
\end{array}\right)(-1)^{k}(k)_{m, \lambda},
$$

where $m \in \mathbb{N} \cup\{0\}$ and $n \in \mathbb{N}$.

For example,

$$
\begin{aligned}
& H_{0, \lambda}^{(1)}(n)=2^{n}, \quad H_{1, \lambda}^{(1)}(n)=n 2^{n-1}, \quad H_{2, \lambda}^{(1)}(n)=n(n+1-2 \lambda) 2^{n-2}, \\
& H_{3, \lambda}^{(1)}(n)=(n)_{3} 2^{n-3}+3(n)_{2}(1-\lambda) 2^{n-2}+n(1-\lambda)_{2, \lambda} 2^{n-1}, \ldots, \\
& T_{0, \lambda}^{(1)}(n)=0, \quad T_{1, \lambda}^{(1)}(n)=-\delta_{1, n}, \\
& T_{2, \lambda}^{(1)}(n)=n(n-1) \delta_{2, n}+(\lambda-1) \delta_{n, 1}, \ldots,
\end{aligned}
$$

where $\delta_{n, k}$ is Kronecker's symbol.

From (49), we note that

$$
\begin{aligned}
\sum_{m=0}^{\infty} T_{m, \lambda}^{(1)}(n) \frac{t^{m}}{m !} & =F^{(1,0)}\left(\begin{array}{c}
-n \\
\times
\end{array} \mid e_{\lambda}(t)\right)=\left(1-e_{\lambda}(t)\right)^{n}=(-1)^{n} \frac{n !}{n !}\left(e_{\lambda}(t)-1\right)^{n} \\
& =(-1)^{n} n ! \sum_{m=n}^{\infty} S_{2, \lambda}(m, n) \frac{t^{m}}{m !} .
\end{aligned}
$$

On the other hand,

$$
\left(1-e_{\lambda}(t)\right)^{n}=\sum_{j=0}^{n}\left(\begin{array}{l}
n \\
j
\end{array}\right)(-1)^{n-j} e_{\lambda}^{j}(t)=\sum_{m=0}^{\infty}\left(\sum_{j=0}^{n}\left(\begin{array}{l}
n \\
j
\end{array}\right)(-1)^{n-j}(j)_{m, \lambda}\right) \frac{t^{m}}{m !} .
$$

Thus, by (54) and (55), we get

$$
T_{m, \lambda}^{(1)}(n)=\sum_{j=0}^{n}\left(\begin{array}{l}
n \\
j
\end{array}\right)(-1)^{n-j}(j)_{m, \lambda}=(-1)^{n} n ! S_{2, \lambda}(m, n) \quad(m \geq n) .
$$

If $m<n$, then $T_{m, \lambda}^{(1)}(n)=0$.

Theorem 3.1 For $n \in \mathbb{N}$ and $m \in \mathbb{N} \cup\{0\}$, we have

$$
T_{m, \lambda}^{(1)}(n)=\sum_{j=0}^{n}\left(\begin{array}{l}
n \\
j
\end{array}\right)(-1)^{n-j}(j)_{m, \lambda}=(-1)^{n} n ! S_{2, \lambda}(m, n) \quad(m \geq n) .
$$


In particular, if $m<n$, then

$$
T_{m, \lambda}^{(1)}=0 .
$$

For $k \geq 0$, we have

$$
\lim _{\lambda \rightarrow 0}(-1)^{n} T_{n+k, \lambda}^{(1)}(n)=\sum_{j=0}^{n}\left(\begin{array}{l}
n \\
j
\end{array}\right)(-1)^{j} j^{n+k}=n ! S_{2}(n+k, n) .
$$

Corollary 3.2 For $k \geq 0$ and $n \in \mathbb{N}$, we have

$$
\frac{1}{n !} \sum_{j=0}^{n}\left(\begin{array}{l}
n \\
j
\end{array}\right)(-1)^{j} j^{n+k}=S_{2}(n+k, n)
$$

It is easy to show that

$$
\begin{aligned}
\left(e^{t}-1\right)^{n} & =\sum_{j=0}^{n}\left(\begin{array}{l}
n \\
j
\end{array}\right)(-1)^{n-j} e^{j t} \\
& =\sum_{j=0}^{n} \sum_{l=0}^{j}\left(\begin{array}{l}
n \\
j
\end{array}\right)(-1)^{n-j}(j)_{l} \frac{1}{l !}\left(e^{t}-1\right)^{l} \\
& =\sum_{j=0}^{n} \sum_{l=0}^{j}\left(\begin{array}{l}
n \\
j
\end{array}\right)(j)_{l}(-1)^{n-j} \sum_{m=l}^{\infty} S_{2}(m, l) \frac{t^{m}}{m !} .
\end{aligned}
$$

Thus, we have

$$
\sum_{m=n}^{\infty} S_{2}(m, n) \frac{t^{m}}{m !}=\frac{1}{n !}\left(e^{t}-1\right)^{n}=\frac{1}{n !} \sum_{j=0}^{n} \sum_{l=0}^{j}\left(\begin{array}{l}
n \\
j
\end{array}\right)(j)_{l}(-1)^{n-j} \sum_{m=l}^{\infty} S_{2}(m, l) \frac{t^{m}}{m !} .
$$

For $k \geq 0, n \in \mathbb{N}$, by (57), we get

$$
S_{2}(n+k, n)=\frac{1}{n !} \sum_{j=0}^{n} \sum_{l=0}^{n+k}\left(\begin{array}{l}
n \\
j
\end{array}\right)(j)_{l}(-1)^{n-j} S_{2}(n+k, l) .
$$

From Corollary 3.2 and (58), we have

$$
\sum_{j=0}^{n}\left(\begin{array}{l}
n \\
j
\end{array}\right)(-1)^{j} j^{n+k}=\sum_{j=0}^{n} \sum_{l=0}^{n+k}\left(\begin{array}{l}
n \\
j
\end{array}\right)(j)_{l}(-1)^{n-j} S_{2}(n+k, l)
$$

For $\lambda, \lambda_{1} \in \mathbb{R}$, let us define Apostol type alternating $\lambda$-hypergeometric numbers of order $p$ by

$$
F^{(p, p-1)}\left(\begin{array}{c}
-n,-n, \ldots,-n \\
1, \ldots, 1
\end{array} \mid(-1)^{p-1} \lambda_{1} e_{\lambda}(t)\right)=\sum_{m=0}^{\infty} T_{m, \lambda}^{(p)}\left(n \mid \lambda_{1}\right) \frac{t^{m}}{m !} .
$$


By (10), we get

$$
\begin{aligned}
F^{(1,0)}\left(\begin{array}{c}
-n \\
\times
\end{array} \mid \lambda_{1} e_{\lambda}(t)\right) & =\sum_{k=0}^{n}\left(\begin{array}{l}
n \\
k
\end{array}\right)\left(-\lambda_{1} e_{\lambda}(t)\right)^{k}=\sum_{j=0}^{n}\left(\begin{array}{l}
n \\
j
\end{array}\right)(-1)^{j} \lambda_{1}^{j} e_{\lambda}^{j}(t) \\
& =\sum_{m=0}^{\infty}\left(\sum_{j=0}^{n}\left(\begin{array}{l}
n \\
j
\end{array}\right)(-1)^{j} \lambda_{1}^{j}(j)_{m, \lambda}\right) \frac{t^{m}}{m !} .
\end{aligned}
$$

On the other hand,

$$
\begin{aligned}
F^{(1,0)}\left(\begin{array}{c}
-n \\
\times
\end{array} \mid \lambda_{1} e_{\lambda}(t)\right) & =\left(1-\lambda_{1} e_{\lambda}(t)\right)^{n}=\sum_{j=0}^{n}\left(\begin{array}{l}
n \\
j
\end{array}\right)(-1)^{j} \lambda_{1}^{j} e_{\lambda}^{j}(t) \\
& =\sum_{j=0}^{n}\left(\begin{array}{c}
n \\
j
\end{array}\right)(-1)^{j} \lambda_{1}^{j} \sum_{l=0}^{j}\left(\begin{array}{l}
j \\
l
\end{array}\right)\left(e_{\lambda}(t)-1\right)^{l} \\
& =\sum_{j=0}^{n} \sum_{l=0}^{j}\left(\begin{array}{l}
n \\
j
\end{array}\right)(-1)^{j} \lambda_{1}^{j}(j)_{l} \frac{\left(e_{\lambda}(t)-1\right)^{l}}{l !} \\
& =\sum_{j=0}^{n} \sum_{l=0}^{j}\left(\begin{array}{c}
n \\
j
\end{array}\right)(-1)^{j} \lambda_{1}^{j}(j)_{l} \sum_{m=l}^{\infty} S_{2, \lambda}(m, l) \frac{t^{m}}{m !} .
\end{aligned}
$$

For $k \geq 0$ and $n \in \mathbb{N}$, by (59), (60), and (61), we obtain the following theorem.

Theorem 3.3 For $\lambda, \lambda_{1} \in \mathbb{R}, n \in \mathbb{N}$, and $k \in \mathbb{N} \cup\{0\}$, we have

$$
T_{n+k, \lambda}^{(1)}\left(n \mid \lambda_{1}\right)=\sum_{j=0}^{n}\left(\begin{array}{l}
n \\
j
\end{array}\right)(-1)^{j} \lambda_{1}^{j}(j)_{n+k, \lambda}=\sum_{j=0}^{n} \sum_{l=0}^{j}\left(\begin{array}{l}
n \\
j
\end{array}\right)(-1)^{j} \lambda_{1}^{j}(j)_{l} S_{2, \lambda}(n+k, l) .
$$

Note that

$$
\lim _{\lambda \rightarrow 0} T_{n+k, \lambda}^{(1)}\left(n \mid \lambda_{1}\right)=\sum_{j=0}^{n}\left(\begin{array}{c}
n \\
j
\end{array}\right)(-1)^{j} \lambda^{j} j^{n+k}=\sum_{j=0}^{n} \sum_{l=0}^{j}\left(\begin{array}{l}
n \\
j
\end{array}\right)(-1)^{j} \lambda_{1}^{j}(j)_{l} S_{2}(n+k, l) .
$$

For $\lambda_{1} \in \mathbb{R}$, let us define Apstol-Stirling numbers of the second kind as

$$
\frac{1}{n !}\left(\lambda_{1} e^{t}-1\right)^{n}=\sum_{m=0}^{\infty} S\left(m, n \mid \lambda_{1}\right) \frac{t^{m}}{m !} \quad(n \geq 0)
$$

Now, we observe that

$$
\frac{1}{n !}\left(\lambda_{1} e^{t}-1\right)^{n}=\frac{1}{n !} \sum_{j=0}^{n}\left(\begin{array}{c}
n \\
j
\end{array}\right) \lambda_{1}^{j}(-1)^{n-j} e^{j t}=\frac{1}{n !} \sum_{j=0}^{n}\left(\begin{array}{c}
n \\
j
\end{array}\right) \lambda_{1}^{j}(-1)^{n-j} \sum_{m=0}^{\infty} j^{m} \frac{t^{m}}{m !}
$$

For $k \in \mathbb{N} \cup\{0\}$ and $n \in \mathbb{N}$, by (62) and (63), we get

$$
S\left(n+k, n \mid \lambda_{1}\right)=\frac{(-1)^{n}}{n !} \sum_{j=0}^{n}\left(\begin{array}{l}
n \\
j
\end{array}\right) \lambda_{1}^{j}(-1)^{j} j^{n+k}
$$


From Theorem 3.3 and (64), we have

$$
\lim _{\lambda \rightarrow 0} T_{n+k, \lambda}^{(1)}\left(n \mid \lambda_{1}\right)=(-1)^{n} n ! S\left(n+k, n \mid \lambda_{1}\right)=\sum_{j=0}^{n}\left(\begin{array}{l}
n \\
j
\end{array}\right) \lambda_{1}^{j}(-1)^{j} j^{n+k} .
$$

Therefore, by (65), we obtain the following corollary.

Corollary 3.4 For $k \in \mathbb{N} \cup\{0\}$ and $n \in \mathbb{N}$, we have

$$
\sum_{j=0}^{n}\left(\begin{array}{l}
n \\
j
\end{array}\right) \lambda_{1}^{j}(-1)^{j} j^{n+k}=(-1)^{n} n ! S(n+k, n \mid \lambda)=\lim _{\lambda \rightarrow 0} T_{n+k, \lambda}^{(1)}\left(n \mid \lambda_{1}\right) .
$$

\section{Remarks}

(a) Corollary 3.4 naturally interprets the sum in Corollary 3.4 in terms of Apostol-Stirling numbers of the second kind defined by (62).

(b) For $\lambda, \lambda_{1} \in \mathbb{R}$, let us define Apostol type $\lambda$-hypergeometric numbers of order $p$ by

$$
F^{(p, p-1)}\left(\begin{array}{c}
-n,-n, \ldots,-n \\
1, \ldots, 1
\end{array} \mid(-1)^{p} \lambda_{1} e_{\lambda}(t)\right)=\sum_{m=0}^{\infty} H_{m, \lambda}^{(p)}\left(n \mid \lambda_{1}\right) \frac{t^{m}}{m !}
$$

By (10), we get

$$
\begin{aligned}
F^{(p, p-1)}\left(\begin{array}{c}
-n,-n, \ldots,-n \\
1, \ldots, 1
\end{array} \mid(-1)^{p} \lambda_{1} e_{\lambda}(t)\right) & =\sum_{k=0}^{\infty}\left(\begin{array}{l}
n \\
k
\end{array}\right)^{p} \lambda_{1}^{k} e_{\lambda}^{k}(t) \\
& =\sum_{m=0}^{\infty}\left(\sum_{k=0}^{n}\left(\begin{array}{l}
n \\
k
\end{array}\right)^{p} \lambda_{1}^{k}(k)_{m, \lambda}\right) \frac{t^{m}}{m !} .
\end{aligned}
$$

From (66) and (67), we have

$$
H_{m, \lambda}^{(p)}\left(n \mid \lambda_{1}\right)=\sum_{k=0}^{n}\left(\begin{array}{l}
n \\
k
\end{array}\right)^{p} \lambda_{1}^{p}(k)_{m, \lambda}, \quad \text { where } n, p \in \mathbb{N} \text { and } m \in \mathbb{N} \cup\{0\}
$$

Note that

$$
\lim _{\lambda \rightarrow 0} H_{m, \lambda}^{(p)}\left(n \mid \lambda_{1}\right)=\sum_{k=0}^{n}\left(\begin{array}{l}
n \\
k
\end{array}\right)^{p} \lambda_{1}^{p} k^{m}
$$

\section{Conclusion}

In this paper, we studied certain finite sums involving powers of binomial coefficients which are called generalized $p$ th order Franel numbers and can be represented in terms of hypergeometric functions. Then, among other things, we found that particular cases of these numbers are connected with many known special numbers and polynomials which include Bernoulli numbers, Euler numbers, Changhee numbers, Daehee numbers, Stirling numbers of the first kind, Catalan numbers, and Legendre polynomials. Recently, Dolgy and Kim gave some explicit formulas of degenerate Stirling numbers associated with the degenerate special numbers and polynomials. Motivated by Dolgy and Kim's paper [4], we 
investigated degenerate versions of the generalized $p$ th order Franel numbers. In more detail, we introduced degenerate generalized hypergeometric functions and studied degenerate hypergeometric numbers of order $p$. We showed that the degenerate hypergeometric numbers of order $p$ involve powers of $\lambda$-binomial coefficients and $\lambda$-falling sequence, and can be represented by means of the degenerate generalized hypergeometric functions. We also considered several related special numbers like $\lambda$-hypergeometric numbers of order $p$ and Apostol type $\lambda$-hypergeometric numbers of order $p$, of which the latter reduce in a limiting case to the generalized $p$ th order Franel numbers.

\section{Acknowledgements}

The authors would like to thank the anonymous reviewers for their valuable comments and suggestions which helped us improve the quality of our work.

\section{Funding}

This work was supported by the National Research Foundation of Korea (NRF) grant funded by the Korea government (MEST) (No. 2017R1E1A1A03070882).

Availability of data and materials

Not applicable.

\section{Competing interests}

The authors declare no conflict of interest.

\section{Authors' contributions}

TK and DSK conceived of the framework and structured the whole paper; DSK and TK wrote the paper; JK paid the article processing charge for the paper; HL typed the paper; DSK and TK completed the revision of the article. All authors have read and agreed to the published version of the manuscript.

\section{Author details}

'School of Science, Xian Technological University, Xian, China. ${ }^{2}$ Department of Mathematics, Kwangwoon University, Seoul, Republic of Korea. ${ }^{3}$ Department of Mathematics, Sogang University, Seoul, Republic of Korea. ${ }^{4}$ Department of Mathematics Education and ERI, Gyeongsang National University, Jinju, Republic of Korea.

\section{Publisher's Note}

Springer Nature remains neutral with regard to jurisdictional claims in published maps and institutional affiliations.

Received: 2 January 2020 Accepted: 6 March 2020 Published online: 12 March 2020

\section{References}

1. Agarwal, P., Younis, J.A., Kim, T.: Certain generating functions for the quadruple hypergeometric series $K_{10}$. Notes Number Theory Discrete Math. 25(4), 16-23 (2019)

2. Carlitz, L.: A degenerate Staudt-Clausen theorem. Arch. Math. (Basel) 7, 28-33 (1956)

3. Carlitz, L.: Degenerate Stirling, Bernoulli and Eulerian numbers. Util. Math. 15, 51-88 (1979)

4. Dolgy, D.V., Kim, T.: Some explicit formulas of degenerate Stirling numbers associated with the degenerate special numbers and polynomials. Proc. Jangjeon Math. Soc. 21(2), 309-317 (2018)

5. Golombek, R.: Aufgabe 1088. Elem. Math. 49, 126-127 (1994)

6. Golombek, R., Marburg, D.: Aufgabe 1088, Summen mit Quadraten von Binomialkoeffizienten. Elem. Math. 50, 125-131 (1995)

7. Haroon, H., Khan, W.A.: Degenerate Bernoulli numbers and polynomials associated with degenerate Hermite polynomials. Commun. Korean Math. Soc. 33(2), 651-669 (2018)

8. He, Y., Araci, S.: Sums of products of Apostol-Bernoulli and Apostol-Euler polynomials. Adv. Differ. Equ. 2014, Article ID 155 (2014)

9. Kim, D.S., Kim, H.Y., Kim, D., Kim, T.: Identities of symmetry for type 2 Bernoulli and Euler polynomials. Symmetry 11 , Article ID 613 (2019)

10. Kim, T.: A note on degenerate Stirling polynomials of the second kind. Proc. Jangjeon Math. Soc. 20(3), 319-331 (2017)

11. Kim, T., Jang, G.-W.: A note on degenerate gamma function and degenerate Stirling number of the second kind. Adv. Stud. Contemp. Math. (Kyungshang) 28(2), 207-214 (2018)

12. Kim, T., Kim, D.S.: Degenerate Laplace transform and degenerate gamma function. Russ. J. Math. Phys. 24(2), 241-248 (2017)

13. Kim, T., Kim, D.S.: A note on type 2 Changhee and Daehee polynomials. Rev. R. Acad. Cienc. Exactas Fís. Nat., Ser. A Mat. 113(3), 2783-2791 (2019)

14. Kim, T., Kim, D.S., Kwon, J.: A note on $\lambda$-hypergeometric random variables. Rev. Educ. 387(2), 49-52 (2020)

15. Kim, T., Yao, Y., Kim, D.S., Jang, G.W.: Degenerate-Stirling numbers and r-Bell polynomials. Russ. J. Math. Phys. 25(1), 44-58 (2018) 
16. Pyo, S.-S., Kim, T.: Some identities of fully degenerate Bell polynomials arising from differential equations. Proc Jangjeon Math. Soc. 22(2), 357-363 (2019)

17. Rainville, E.D.: Special Functions. Chelsea, New York (1971)

18. Rim, S.-H., Kim, T., Lee, S.H.: Some symmetry identities for $(h, q)$-Bernoulli polynomials under the third dihedral group $D_{3}$ arising from $q$-Volkenborn integral on $\mathbb{Z}_{p}$. J. Comput. Anal. Appl. 20(3), 432-436 (2016)

19. Rim, S.-H., Kim, T., Pyo, S.-S.: Identities between harmonic, hyperharmonic and Daehee numbers. J. Inequal. Appl. 2018, Article ID 168 (2018)

20. Roman, S.: The Umbral Calculus. Pure and Applied Mathematics, vol. 111. Academic Press, New York (1984)

21. Whittaker, E.T., Watson, G.N.: Modern Analysis, 4th edn. Cambridge University Press, Cambridge (1927)

22. Zhang, W., Lin, X.: Identities involving trigonometric functions and Bernoulli numbers. Appl. Math. Comput. 334, 288-294 (2018)

Submit your manuscript to a SpringerOpen ${ }^{\odot}$ journal and benefit from:

- Convenient online submission

- Rigorous peer review

- Open access: articles freely available online

- High visibility within the field

- Retaining the copyright to your article

Submit your next manuscript at $\gg$ springeropen.com 